\title{
Hydrokinetic turbine location analysis by a local collocation method with Radial Basis Functions for two-dimensional Shallow Water equations
}

\author{
C. A. Bustamante ${ }^{1}$, W. F. Florez ${ }^{1}$, H. Power ${ }^{2}$ \& A. F. Hill ${ }^{1}$ \\ ${ }^{1}$ School of Engineering, Universidad Pontificia Bolivariana, Colombia \\ ${ }^{2}$ School of Mechanical, Materials and Manufacturing Engineering, \\ University of Nottingham, UK
}

\begin{abstract}
Recently, hydrokinetic turbines arise as a suitable technology for renewable energy production in isolated zones where solar, wind and tidal energy are limited. Hydrokinetic turbines convert kinetic energy of free water flow in rotational kinetic energy. This energy is finally converted to electrical energy by an integrated generator. Regarding the fact that power output is proportional to the cube of river average velocity, knowing velocity distribution in the installation zone is a crucial aspect for an optimum turbine operation. In this study, two-dimensional Shallow Water equations (SWEs) are solved by the meshless method of local collocation with Radial Basis Function in order to obtain the depth velocity average distribution and water height in open channel flow. Firstly, power output is estimated for a conventional turbine located in the centre of a straight channel. Afterwards, SWEs are solved in a channel with elements to increase local velocity with the aim of estimating power output increment. Electricity production can be increased by adding geometrical elements which modify flow pattern keeping an adequate depth for turbine operation.

Keywords: hydrokinetic turbines, radial basis functions, local collocation, openchannel flow, power estimation.
\end{abstract}




\section{Introduction}

Hydrokinetic turbines have arose as a suitable option for renewable energy production in zones where solar and wind energies are limited. Several turbine technologies has been implemented in order to capture kinetic energy from a river, such as horizontal, vertical and transversal axis [1-3]. No matter technology implemented, power coefficients provides the maximum ratio between mechanical power extracted (available shaft work rate) and the kinetic energy rate of the current, being its highest value the betz limit (0.59). In this sense, turbine work rate is function of power coefficient, fluid density, swept-area and the cube of free-current velocity. Power coefficient depends on technology use and turbine geometrical aspects as well as operational conditions. In this case fluid density is constant since turbine emplacing is conceived to be in a river while swept-area depends on the square of characteristic length which is restricted by water depth. Therefore, current-velocity must be an important criteria when planning installation of hydrokinetic turbines since modification of this value will have a crucial impact of power extracted. Recently, river velocity has been artificially increased by using venturi inner shape ducts surrounding the turbine [4]. Nevertheless the impact of this strategy on water depth and river velocity has not been study in detail. A computational model that can predict in a simple manner and with a low computational cost, height and velocity in the river is needed for evaluating the effect of emplacing obstacles to locally increase current velocity. In this work, the Shallow Water Equations (SWEs) are used with this purpose.

SWEs have been widely used to model one- and two-dimensional open-channel flow in situations whereby depth is much smaller than wide or velocity variation along depth is negligible such as rivers, bays and straits. SWEs consists in a system of non-linear equations in terms of depth-average velocity vector and water surface height. Additionally, in most of the practical problems the SWEs must be solved in complex domains given the geometrical irregularities presented in boundaries of open water systems. Therefore, the use of meshless methods for solving SWEs has been popularising during the last decades regarding the reduction in preprocessing time, the use of high-order approximations and a versatile treatment of boundary conditions. Several meshless methods has been applied to the solution of SWEs. Among meshless methods, RBF direct collocation has been used to solve a variety of partial differential equation (PDE) problems such as Poisson, Helmholtz, Convection-diffusion and Navier-Stokes. Given a straightforward implementation and the excellent behaviour of RBF in interpolation, the application of direct RBF collocation for solving SWEs has been popularised recently [5-7].

In this work, a simple localised RBF collocation method is implemented for solving two-dimensional SWEs in open-channel flow with the aim of evaluating the effect on hydrokinetic turbine performance of adding obstacle to the channel flow with the purpose of increasing current velocity at turbine emplacing zone. Firstly, the hydrokinetic turbine technology is presented and performance indicators are shown. Then, SWEs are briefly explained and the numerical meshless method used for the analysis is reviewed. Finally, In the numerical result 
section, one- and two-dimensional validation problems are presented and results for different obstacle configuration are shown in order to discuss its effect on turbine mechanical power output.

\section{Hydrokinetic turbines}

Hydrokinetic turbines are employed to extract kinetic energy from a water current by emplacing a rotor into the flow. Common application are the extraction energy from tides and rivers. As wind turbines, hydrokinetic devices can be classified into two major groups: axial and cross flow turbines. Propeller-type turbines belong to the first group regarding the fact that axis is parallel to main stream velocity. It is suitable for being utilised in rivers where direction of flow is almost constant. Nevertheless, there are some disadvantages such as high impact of velocity fluctuations on performance, use of high-resistant material to resist debris flow and the need of submersible generator [3]. Some of this issues can be avoided by using the cross flow technology, such as Savonius, Darrieus and Gorlov turbines (for more information see [2]), because axis is perpendicular to the river bed allowing the generator to be placed up to the water level. Unlike Darrieus and Gorlov technologies, Savonius turbine is a drag-type device. Additionally, lower noise level, less sensibility to flow fluctuations, less effect of depth-velocity profile on its performance make vertical axis turbine a suitable option. Also, turbines with horizontal axis perpendicular to flow direction and parallel to water surface are considered as cross flow type turbines. No matter the turbine type, performance can be expressed in terms of power coefficient $C_{P}$ which relates power output $P$ to energy kinetic rate of current through area swept by turbine blades $A$, as follows

$$
P=\frac{1}{2} C_{P} \rho A U_{\infty}^{3}
$$

where $\rho$ and $U_{\infty}$ are fluid density and average free current velocity. Maximum value of $C_{P}$ is 0.59 or the Betz limit. Values reported for wind axial turbines are as high as 0.5 while the highest value found for vertical axis is 0.31 measured in a Gorlov turbine [2]. Besides blade geometry and configuration, power coefficient depends on the tip speed ratio given by:

$$
\lambda=\frac{\omega R}{U_{\infty}}
$$

with $R$ as the distance between blade tip and axis. Normally, maximum value of $C_{P}$ is found for a specific $\lambda$.

Several strategies can be implemented in order to increase power output. As shown in equation (1), in case of increasing velocity, power can rise as the cube of velocity provided that $\lambda$ remain almost constant. Duct augmentation is the most common strategy and consists in placing constrains to the flow such as duct, nozzle, concentrator, diffuser, or augmentation channel with the aim of increasing the velocity in zone upstream the turbine [4]. 


\section{Local RBF collocation method for SWEs}

\subsection{Shallow water equations}

The two-dimensional SWEs includes mass balance and the two components of the momentum conservation law, both of them in terms of the water surface height $h$ and depth-average velocity components $u$ and $v$, as follows:

$$
\begin{aligned}
\frac{\partial h}{\partial t}+\frac{\partial h u}{\partial x}+\frac{\partial h v}{\partial y} & =0 \\
\frac{\partial h u}{\partial t}+\frac{\partial h u^{2}}{\partial x}+\frac{\partial h u v}{\partial y}+g h \frac{\partial H}{\partial x} & =-g h S_{f_{x}} \\
\frac{\partial h v}{\partial t}+\frac{\partial h u v}{\partial x}+\frac{\partial h v^{2}}{\partial y}+g h \frac{\partial H}{\partial y} & =-g h S_{f_{y}}
\end{aligned}
$$

Water surface height $h$ is measured from soil bottom, whose shape is given by the topography function $z(x, y)$, while total height is computed as $H=h+z$. In this case, the friction force $\overrightarrow{S_{f}}=\left(S_{f_{x}}, S_{f_{y}}\right)$ is expressed according to the Manning-Striker's law, in the following way:

$$
\overrightarrow{S_{f}}=n^{2} \frac{\vec{U} \sqrt{u^{2}+v^{2}}}{h^{\frac{3}{4}}}
$$

where $\vec{U}=(u, v)$ and $n$ is the Manning constant. Three types of boundary conditions are commonly used in SWEs: known height, known $x$ and $y$ discharge ( $u h$ and $v h$, resp.) and land. In case of land boundary condition $u=0$ and $v=0$.

\subsection{RBF collocation method}

As Kansa [8] formulates, the dependent variable of a boundary value problem can be approximated as a lineal combination of RBFs. When the approximation is substituted into de governing PDE and the boundary condition and the resulting expression is evaluated in a finite number of nodes scattered throughout domain and boundary, it is possible to obtain a linear system of equations whose solution are the coefficients of the original lineal combination of RBFs. Regarding the fact that SWEs are a set of non-linear PDEs, RBF collocation must be implemented in conjunction to a linearisation scheme or a non-linear solver. In our case, the Newton-Raphson method is implemented and an implicit scheme is applied for time discretisation.

Let us consider a generic function $\phi$ which is approximated by RBFs, in this case the Multiquadric (MQ) function with $m=1, \psi=\left(r^{2}+c^{2}\right)^{1 / 2}$ as follows:

$$
\phi=\sum_{i=1}^{N} \alpha_{i} \psi\left(r_{i}\right)+\sum \beta P(\vec{x})_{k}^{m-1}
$$


with $N$ as the number of points $\vec{\epsilon}$ whereby the RBF is evaluated, regarding that $r_{i}=\sqrt{\left(\vec{x}-\vec{\epsilon}_{i}\right) \bullet\left(\vec{x}-\vec{\epsilon}_{i}\right)}$. In the localised formulation, $N$ is the number of the nearest points to the collocation point $\vec{x}$, i.e. nodes inside of the subdomain defined by a characteristic number of nodes or local radius. The polynomial term is added in order to ensure invertibility of resulting interpolation matrix. Given $m=1$, the polynomial terms are reduced to a single constant. Therefore, $\phi$ spatial derivatives are calculated according to:

$$
\frac{\partial \phi}{\partial x_{k}}=\sum_{i=1}^{N} \alpha_{i} \frac{\partial \psi\left(r_{i}\right)}{\partial x_{k}}
$$

After collocation of equation (7) at the nodes located inside a subdomain, linear system of equations is obtained and unknown coefficients can be expressed in terms of unknown variable values as $[\alpha]=[\Psi]^{-1}[\Phi]$ with $[\Psi]$ and $[\Phi]$ as the collocation matrix and the column vector of nodal values of variable $\Phi$, respectively. Therefore, equation (7) is rewritten in terms of unknown variable values and matrix products, as:

$$
\phi(\vec{x})=[G(\vec{x})][\Psi]^{-1}[\Phi]
$$

where $1 \times N+1$ matrix $[G(\vec{x})]=\left[\phi\left(r_{1}\right), \cdots, \phi\left(r_{N}\right), 1\right]$.

With the aim of avoiding discontinuity effects on the stability of the solution, a slightly different approximation is done here, in the sense that velocity and height derivatives are not approximated by RBFs but the spatial derivatives of variable products according to the governing equations. In order to discretise SWEs, the generic variable $\phi_{j}$ takes the values reported in Table 1 in terms of the index $j$.

Table 1: Values of generic variable $\phi$.

\begin{tabular}{c|cccccc}
\hline$j$ & 1 & 2 & 3 & 4 & 5 & 6 \\
\hline$\phi_{j}$ & $h u$ & $h v$ & $h u^{2}$ & $h v^{2}$ & $h u v$ & $H$ \\
\hline
\end{tabular}

If the RBF approximations are substituted into equations from (3) to (5) and the temporal derivatives are approximated by an implicit first-order scheme, a set of discretised non-linear algebraic equations, corresponding to the generic case of an interior subdomain including $N$ nodes, is obtained. After applying those equations to each one of the internal nodes and, by replacing the corresponding one with the boundary condition, at boundary nodes, a non-linear system of $3 N_{t}$ equations, with $N_{t}$ as the sum of internal and boundary nodes, is obtained. In the present case, the system of equation is solved by using the Newton-Raphson method for each time step. 


\section{Numerical results}

One and two dimensional shallow water problems with analytical solution are solved in order to validate the implemented scheme. Accuracy of spatial discretisation is evaluated for the 1-D problem of flow over a bump. Spatial discretisation in 2-D situations is assessed by solving a transient problem with analytical solution. In the last part of this section, methodology for estimation of power output for a turbine emplaced in an open channel with and without constrains are presented. Additionally, effect of constrains in power output is discussed.

\subsection{1-D problems}

Different analytical solutions of SWEs for one-dimensional problems can be obtained according to the methodology presented in [9] after defining the topography function $z(x, y)$ and the friction term value. With the aim of testing the accuracy of the spatial discretisation, the transient code is employed to solve the steady problems starting at an initial guess. As a validation problem, the steady and frictionless flow in a one-dimensional open channel with a bump is addressed. The domain length is $L=25$ and the topography function is given by:

$$
z(x)=\left\{\begin{array}{lc}
0.2-0.05(x-10)^{2} & 8<x<12, \\
0 & \text { else }
\end{array}\right.
$$

Given the topography function (10), the following analytical solution is reported for height in [9]:

$$
h(x)^{3}+\left(z(x)-\frac{p_{0}^{2}}{2 g h_{L}{ }^{2}}-h_{L}\right) h(x)^{2}+\frac{p_{0}^{2}}{2 g}=0
$$

where $p_{0}$ is a constant value for the discharge, fixed as a boundary condition, as well as the height at outflow boundary given by $h_{L}=h(L)$. The initial solution corresponds to water at rest, i.e. $h(x)+z(x)=2$ and $h(x) u(x)=p(x)=0$ at $t=0$. Constant values for boundary conditions are given by $h(0) u(0)=$ $p_{0}=4.42$ and $h(L)=2$. Once analytical height is obtained by solving the third grade polynomial (11), it is possible to find velocity since discharge $p=h u$ is constant throughout domain for one-dimensional steady situations (see equation (3)). Topography shows a bump with a maximum at $x=10$. The bump produces a small depression on the surface and a corresponding velocity increase. Downwind the bump, velocity and height values are the same as at the inlet boundary.

With the aim of validating the method and analysing the convergence order of the spatial discretisation scheme, the present problem is solved for different number of nodes and different values of the shape parameter $c$ of the MQ function (Figure 1). In the first case, four nodal distribution are employed $(N=41,81,161$, 321 ) and, according to trend obtained, the convergence of the scheme is shown. 

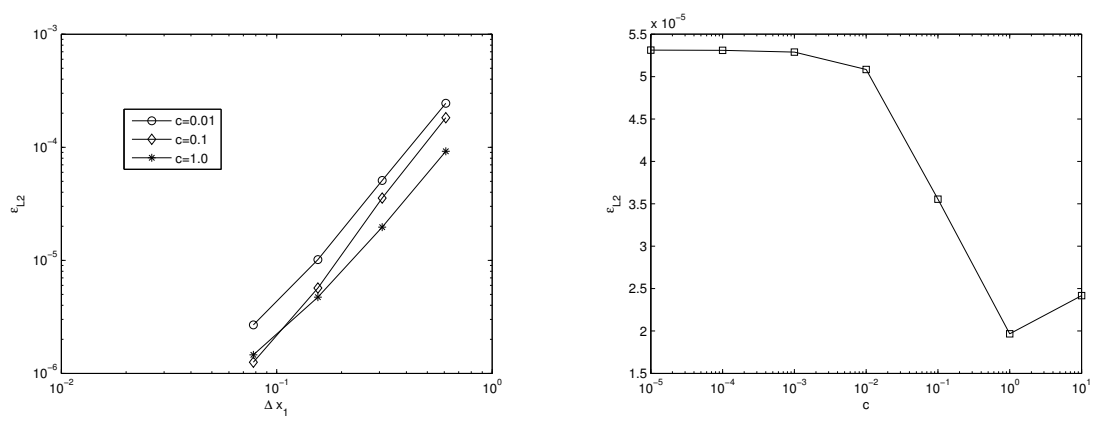

Figure 1: Relative $L_{2}$ norm of the height error in terms of node spacing (left) and shape parameter (right) for subcritical flow over a bump.

If a potential regression is made with data presented in Figure 1, a second-order convergence rate is found (between 2.02 and 2.44). The error behaviour in terms of the shape parameter agrees the usual trend in direct RBF collocation methods: after a constant value zone, the error decreases when the shape parameter increases up to a critical value whereby the error increases due to the ill-conditioning of the local interpolation matrices.

\subsection{2-D problem}

Different two-dimensional analytical solutions for the SWEs were obtained by Thacker [10]. Here, the radially symmetrical oscillating paraboloid problem, as presented in [9], is solved by using the meshless scheme developed. The topography is a paraboloid defined by the function $z(r)=-h_{0}\left(1-\frac{r^{2}}{a^{2}}\right)$, where $h_{0}$ is the depth at central point of the domain when $H=0$ (zero elevation), $a$ is the distance between central point and zero elevation circle in the shoreline and $r$ is the Euclidian distance from central point to any point $(x, y)$ in the domain $[0, L] \times[0, L]$.

The periodic solution for the frictionless flow case, is given by:

$$
\begin{gathered}
h(r, t)=h_{0}\left[\frac{\sqrt{1-A^{2}}}{1-A \cos \omega t}-1-\frac{r^{2}}{a^{2}}\left(\frac{1-A^{2}}{(1-A \cos \omega t)^{2}}-1\right)\right]-z(r) \\
u(x, y, t)=\frac{1}{1-A \cos \omega t}\left[\frac{1}{2} \omega\left(x-\frac{L}{2}\right) A \sin \omega t\right] \\
v(x, y, t)=\frac{1}{1-A \cos \omega t}\left[\frac{1}{2} \omega\left(y-\frac{L}{2}\right) A \sin \omega t\right]
\end{gathered}
$$



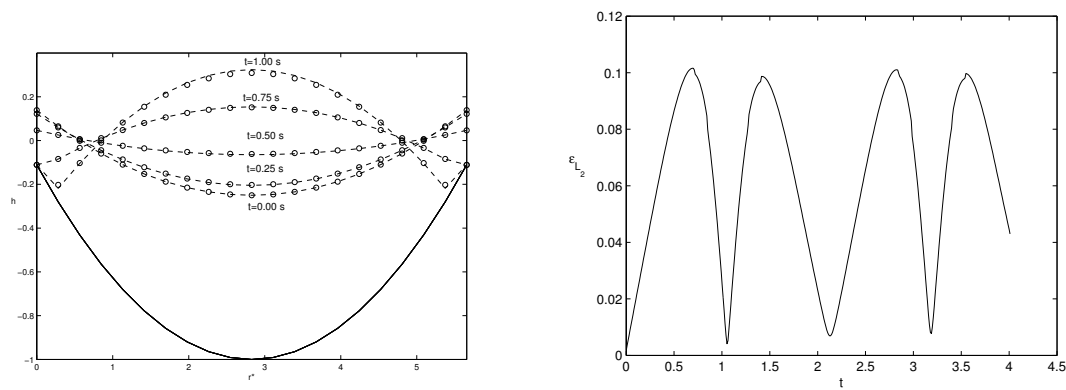

Figure 2: Numerical (circles) and analytical (continuous line) height solution of the radially-symmetrical paraboloid problem (left) and Relative $L-2$ norm of the height error for a simulation time of $4 \mathrm{~s}$ (right).

where $\omega=\sqrt{8 g h_{0}} / a$ is the frequency, $r_{0}$ is the distance between central point and the initial position of shoreline and $A=\left(a^{2}-r_{0}^{2}\right) /\left(a^{2}+r_{0}^{2}\right)$. In order to estimate boundary conditions from analytical solution, the following values of solution parameters are considered: $a=3, r_{0}=4, h_{0}=1$ and $L=4$. Transient numerical solution is attained for half oscillation period given by $T=\frac{\pi}{\omega}$ and by fixing the following numerical parameters: $c=10, n=8$ and $\Delta t=0.01$. Nodal distribution consists in a grid of $N=21 \times 21$ points equally spaced throughout the square domain.

The numerical solution obtained for height along line $y=x$ are presented in Figure 2 for different times, with $r *=\sqrt{x^{2}+y^{2}}$. The solution is in good agreement with analytical function for the period of simulation. Nevertheless, error increases as time passes but given the oscillatory nature of the problem the error reduces once an oscillation is completed as shown in right part of Figure 2 where relative $L_{2}$-norm error of height are presented for a greater time of simulation $(t=4 s)$.

Although it is not shown for brevity, the two components of velocity exhibit similar behaviour than height but relative error are higher towards boundary at $t=1.0$ due to the presence of a wet-dry interphase. However, the numerical results attained for the solution of this problems shows applicability of the implemented method to solve two-dimensional flow situations.

\subsection{Open channel flow: hydrokinetic power increment}

First of all, consider a straight open channel with a length-wide ratio of $L / D=5$ and plane topography, i.e. $z=0$. Inlet velocity boundary condition (at $x_{1}=0$ ) is $u=0.5$ and $v=0$ while outflow condition (at $x=L$ ) corresponds to fixed water height $h=0.5$. Lateral boundaries $\left(x_{2}=0\right.$ and $\left.x_{2}=D\right)$ are 

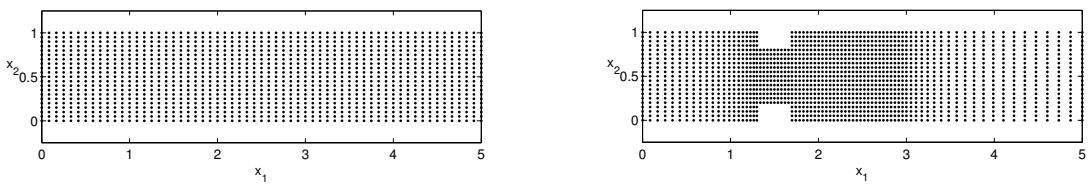

Figure 3: Nodal distribution for straight channel (left) and for channel with obstacles (right).
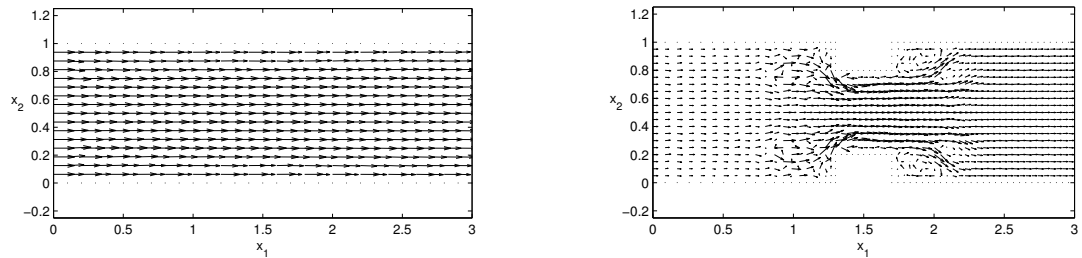

Figure 4: Velocity field for straight channel (left) and for channel with obstacles (right).

considered walls (zero-velocity). Figure 3 (up) shows the homogeneous nodal distribution employed. In order to compare hydrokinetic power increasing with respect straight channel, a second computational domain is proposed with similar boundary condition but obstacles of $0.2 \times 0.4$ size placed symmetrically. With the aim of capturing vortex formation close to the obstacles, nodal distribution is refined towards those zones as it is shown in Figure 3 (down).

Suppose that an axial hydrokinetic turbine of diameter $D_{T}=0.2$ is emplaced in the point $(0.5,1.5)$. If power coefficient is supposed to be constant for a wide range of tip speed ratios and available power is estimated with equation (1), the increasing in power output is given by:

$$
I=\frac{P_{2}}{P_{1}}=\frac{{\overline{u_{2}}}^{3}}{{\overline{u_{1}^{3}}}^{3}}
$$

where subscript 1 is for straight channel and 2 for channel with obstacle. Average velocity in turbine emplacing zone is computed, numerically, as:

$$
\bar{u}=\frac{1}{D_{T}} \int_{1 / 2 *\left(1-D_{T}\right)}^{1 / 2 *\left(1+D_{T}\right)} u d x_{2}
$$

Velocity field obtained when solution reaches steady state are presented in Figure 4. Fluid is accelerated when passing between the obstacles as it was 


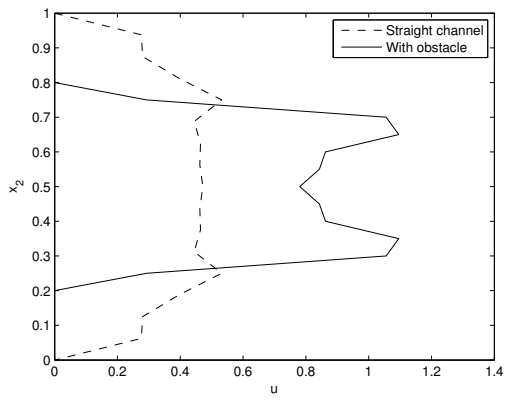

Figure 5: $x_{1}$-Velocity profile in $x_{1}=1.5$.

expected. Velocity profiles in the turbine emplacing line $\left(x_{1}=1.5\right)$, for both cases, are shown in Figure 5. Increment on velocity average near turbine zone in the channel with obstacles is equivalent to a power output increment of 7.51 times the value in the straight channel case.

\section{Conclusions}

A methodology for estimating the effect of morphological changes in open channel on hydrokinetic power output has been developed based on the local collocation method with Radial Basis Functions for solving Shallow Water Equations. This methodology could be used to predict power output for different channel geometries and turbine positions. In the example presented, an increasing of 7.51 times in power is estimated by adding symmetric obstacles to a straight channel. Future work will be focused on improved spatial discretisation to addressed more complex geometries and supercritical regime flow.

\section{References}

[1] Anyi, M. and Kirke, B., Evaluation of small axial flow hydrokinetic turbines for remote communities. Energy for Sustainable Development, 14, pp. 110$116,2010$.

[2] Guney, M.S. and Kaygusuz, K., Hydrokinetic energy conversion systems: A technology status review. Renewable and Sustainable Energy Reviews, 14, pp. 2996-3004, 2010.

[3] Khan, M., Bhuyan, G., Iqbal, M. and Quaicoe, J., Hydrokinetic energy conversion systems and assessment of horizontal and vertical axis turbines for river and tidal applications: A technology status review. Applied Energy, 86, pp. 1823-1835, 2009.

[4] Guney, M.S., Evaluation and measures to increase performance coefficient of hydrokinetic turbines. Renewable and Sustainable Energy Reviews, 15, pp. 3669-3675, 2011. 
[5] Hon, Y.C., Cheung, K., Mao, X.Z. and Kansa, E., Multiquadric solution for shallow water equations. Journal of Hydraulic Engineering, 125(5), pp. 524533, 1999. Cited By (since 1996)132.

[6] Sun, C.P., Young, D., Shen, L.H., Chen, T.F. and Hsian, C., Application of localized meshless methods to $2 \mathrm{~d}$ shallow water equation problems. Engineering Analysis with Boundary Elements, 37(11), pp. 1339-1350, 2013. Cited By (since 1996)0.

[7] Khoshfetrat, A. and Abedini, M., Numerical modeling of long waves in shallow water using lrbf-dq and hybrid dq/lrbf-dq. Ocean Modelling, 65, pp. 1-10, 2013. Cited By (since 1996)1.

[8] Kansa, E.J., Multiquadrics - a scattered data approximation scheme with applications to computational fluid dynamics-ii solution to parabolic, hyperbolic and elliptic partial differential equations. Comput Math Appl, 19(8-9), pp. 127-145, 1990.

[9] Delestre, O., Lucas, C., Ksinant, P.A., Darboux, F., Laguerre, C., Vo, T.N.T., James, F. and Cordier, S., Swashes: A compilation of shallow water analytic solutions for hydraulic and environmental studies. International Journal for Numerical Methods in Fluids, 72(3), pp. 269-300, 2013. Cited By (since 1996)3.

[10] Thacker, W.C., Some exact solutions to the nonlinear shallow-water wave equations. Journal of Fluid Mechanics, 107, pp. 499-508, 1981. Cited By (since 1996)168. 